\section{Projection Method for Exact Diagonalization in the Strong Coupling Limit for Frustrated Models with Highly Degenerate Ground States}

\author{
Keisuke Matsuda $^{1}$, Shin Miyahara ${ }^{2}$, and Nobuo \\ FURUKAWA $^{1,2}$ \\ ${ }^{1}$ Department of Physics and Mathematics, Aoyama Gakuin \\ University, Sagamihara, Kanagawa 229-8558 \\ ${ }^{2}$ Multiferroics Project (MF), ERATO, Japan Science and \\ Technology Agency (JST), c/o Department of Applied \\ Physics, The University of Tokyo, Tokyo 113-8656
}

KEYWORDS: geometrical frustration, projection, exact diagonalization, $t-V$ model, $\mathrm{XXZ}$ Heisenberg model

Numerical calculations play an important role in clarifying the features in strongly correlated electron systems. ${ }^{1}$ In particular, quantum Monte Carlo (QMC) and density matrix renormalization group (DMRG) methods are often adopted to quantum spin and electron systems. However, most of them are useful only for the system without geometrical frustration or in low dimension.

Exact diagonalization (ED) is applicable even in twoor three-dimensional frustrated systems, although there exist limitations to system sizes, e.g., 36 sites in twodimensional spin-1/2 kagomé antiferromagnetic Heisenberg systems at present. To increase the system size, calculations with some approximation have been attempted. One of the successful methods is a numerical contractor renormalization method. ${ }^{2,3}$ In this method, an effective Hamiltonian is constructed on a truncated local basis set with ED and the calculated system size increases up to 48 sites in a spin- $1 / 2$ kagomé system. ${ }^{3}$

In this paper, we carry out a new technique named projection ED and indicate that it is applicable to the frustrated models in the strong coupling limit with highly degenerate ground states in a general way. Let us consider the Hamiltonian

$$
\mathcal{H}=\mathcal{H}_{0}+\mathcal{H}_{1} .
$$

Here, the Hamiltonian $\mathcal{H}_{0}$ has classical degrees of freedom and its eigenvalues are discrete. In addition, its ground state is assumed to be macroscopically degenerate and to have an excitation gap $\Delta$ (see Fig. 1(a)). $\mathcal{H}_{1}$ is a quantum term that hybridizes the eigenstates of $\mathcal{H}_{0}$, which will be treated perturbatively.

As typical examples, we consider frustrated XXZ spin$1 / 2$ Heisenberg and spinless fermion $t-V$ models in a strong coupling region, i.e., $\left|J_{x y}\right| \ll J_{z}$ in the former model and $|t| \ll V$ in the latter model, where $J_{x y}$ and $t$ give perturbation terms $\mathcal{H}_{1}$. The term $\mathcal{H}_{0}$ corresponds to an Ising model. On some geometrically frustrated lattices, i.e., on triangular, kagomé and pyrochlore lattices, the models with $\mathcal{H}_{1}=0$ have macroscopically degenerate ground states when interactions are antiferromagnetic, i.e., $J_{z}>0$ or $V>0 .^{4-7}$

For $\Delta \gg \varepsilon_{1}$, where $\varepsilon_{1}$ is the energy scale of $\mathcal{H}_{1}$, we may apply the perturbation theory to describe low energy

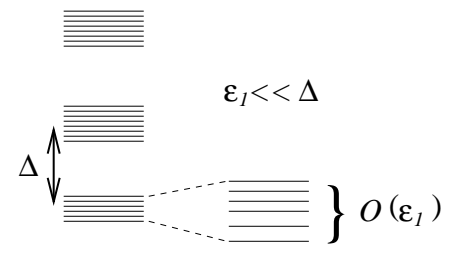

(a)

(b)

Fig. 1. (a) Macroscopic degenerate ground state with excitation energy $\Delta$. (b) Calculated energy within the projected subspace for $\varepsilon_{1} \ll \Delta$.

states as those spanned within the Hilbert space projected to the degenerate ground states of $\mathcal{H}_{0}$, as shown in Fig. 1(b). Within the second order of the perturbation of $\mathcal{H}_{1}$, we can obtain an effective Hamiltonian:

$$
\tilde{\mathcal{H}}=E_{0}+\mathcal{P} \mathcal{H}_{1} \mathcal{P}-\mathcal{P} \mathcal{H}_{1} \mathcal{Q} \frac{1}{\mathcal{H}_{0}} \mathcal{Q} \mathcal{H}_{1} \mathcal{P},
$$

where $\mathcal{P}$ is a projection operator, which takes 1 in case that the applied state is one of the ground states of the Ising model, and 0 for higher energy states. $\mathcal{Q}$ is the complement of $\mathcal{P}$ and satisfies $\mathcal{P}+\mathcal{Q}=1 . E_{0}$ is the ground state energy of the classical model $\mathcal{H}_{0}$.

In these cases, we have difficulties caused by (i) macroscopic degeneracy in the ground state and (ii) the nonlocal property of $\mathcal{P}$. Due to the former reason, analytical approaches for perturbative calculations are extremely difficult. Therefore, we need to resort to numerical calculations. To the advantage of ED, the number of ground states of $\mathcal{H}_{0}$ is much smaller than the total dimension of the original Hilbert space of $\mathcal{H}$, in general. Thus, it becomes possible to handle a much larger system than in the case of conventional ED.

The latter difficulty originates from the fact that, unlike the simple cases such as the Hubbard model at $U \rightarrow \infty$, the unperturbed ground states themselves are highly nontrivial. This gives complex forms for analytical expressions of the projection operators $\mathcal{P}$ and $\mathcal{Q}$, and hence, the overall perturbation terms. In addition, since the ground states depends on lattice structures, the formula also depends on them and is thus nonuniversal.

To avoid such difficulties, we introduce ED, which consequently makes it possible to treat the problem in general. Hereafter, we show the details based on the calculations for the spinless fermion $t-V$ model on a triangular lattice as an example. A Hamiltonian can be written as

$$
\begin{aligned}
& \mathcal{H}_{0}=V \sum_{\langle i j\rangle}\left(n_{i}-\frac{1}{2}\right)\left(n_{j}-\frac{1}{2}\right), \\
& \mathcal{H}_{1}=-t \sum_{\langle i j\rangle}\left(c_{i}^{\dagger} c_{j}+\text { H.c. }\right) .
\end{aligned}
$$

Here, $c_{i}^{\dagger}\left(c_{i}\right)$ is the creation (annihilation) operator of fermions at site $i$ and $n_{i}=c_{i}^{\dagger} c_{i}$. $V$ is a repulsive interaction between nearest neighboring sites. We allow hopping between nearest neighboring sites on a triangular lattice with an amplitude $t>0$.

Lanczos procedure has been performed by applying the Hamiltonian $\mathcal{H}_{1}$, which is an $\mathcal{O}(M)$ calculation. Here, $M$ 


\begin{tabular}{|r|r|r|}
\hline$N$ & \multicolumn{1}{|c|}{$M(\mathrm{ED})$} & $M$ (projection ED) \\
\hline \hline 12 & 924 & 66 \\
\hline 18 & 48,620 & 386 \\
\hline 24 & $2,704,156$ & 2,142 \\
\hline 30 & $155,117,520$ & 13,694 \\
\hline 36 & $9,075,135,300$ & 84,486 \\
\hline 42 & $538,257,874,440$ & 553,574 \\
\hline 48 & $32,247,603,683,100$ & $3,720,042$ \\
\hline
\end{tabular}

Table I. Number of states at half-filling for conventional and projection EDs on $\mathrm{N}$-site clusters for the triangular $t-V$ model.

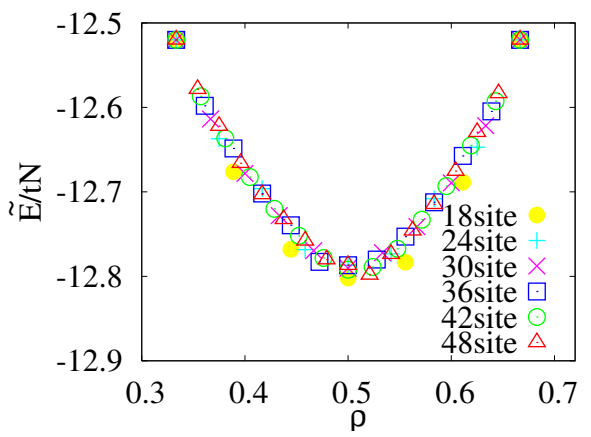

Fig. 2. (Color online) Energies $\tilde{E} / t N$ at density $\rho$ on triangular spinless fermion $t-V$ model for $V / t=50$. The results for up to 48-site clusters are shown.

is the number of ground states of $\mathcal{H}_{0}$ at a given fermion density. For the hopping from site $i$ to $j$, a locally modified energy can be obtained by confirming the 10-site configuration including 8 neighboring sites of $i$ and/or $j$ on a triangular lattice. When the modified state belongs to the ground state of $\mathcal{H}_{0}$, the hopping term contributes as the first-order perturbation term in eq. (2). In the case that the modified state is an excited state of $\mathcal{H}_{0}$, further hopping processes which return the excited state to one of the ground states, lead to the second-order perturbation term in eq. (2). Generally, the possibility of such a correlated hopping can be confirmed by considering the 13 -site configuration at most. Since what we need is the energy of a locally modified state for the Ising model, the type of calculation is an $\mathcal{O}(1)$ calculation. In this manner, projection ED is generally an $\mathcal{O}(M)$ calculation. Note that, even if we got the formula in eq. (2) analytically, its direct Lanczos operation is also $\mathcal{O}(M)$.

As shown in Table I, the number of states $M$ is much smaller than that of conventional ED. Thus, we can reach a much larger cluster than in the case of conventional ED. For example, we could carry out the calculation up to 48-site clusters in the second order of perturbation easily. Energies $\tilde{E} / t$ as a function of fermion densities are shown in Fig. 2. Here, the calculations have been performed for the numbers of sites $N=18,24,30,36,42$ and 48 at the densities $1 / 3 \leq \rho \leq 2 / 3$ for $V / t=50$. Note that we can handle a much larger system on kagomé and pyrochlore lattices by also applying projection ED.

The accuracy of our method can be confirmed by comparison with conventional ED in small clusters. We calculate the energy differences between projection and conventional EDs using $\delta=|(\tilde{E}-E) / E|$, where $\tilde{E}$ is the

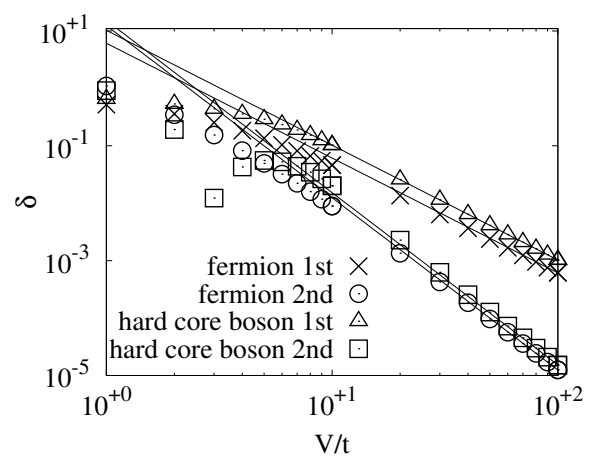

Fig. 3. Comparison between conventional and projection EDs on the spinless fermion $t-V$ and hard core boson models on the triangular lattice. The calculation has been performed at halffilling for both first- and second-order perturbations. For $V / t \gtrsim$ 10 , the energy differences are proportional to $(t / V)^{2}$ for the firstorder calculation and $(t / V)^{3}$ for the second-order calculation. The fitting results are shown by the lines.

energy calculated by projection $\mathrm{ED}$, and $E$ is the energy obtained by ED. The results for a 24 -site cluster are shown in Fig. 3. In the parameter region, where the $n$-th order of perturbation is sufficiently accurate, $\tilde{E}$ is consistent with $E$ within $\mathcal{O}\left((t / V)^{n}\right)$, i.e., the errors $\delta$ are proportional to $(t / V)^{n+1}$. As shown in the figure, we can obtain a good agreement in the second-order perturbation for $V / t \gtrsim 10$, where the errors are proportional to $(t / V)^{3}$. In the figure, the results for a hard core boson model on a triangular lattice are also shown.

Finally, we mention that researches of the hard core boson and $t-V$ models on the triangular lattice by projection ED are significant. These models have been paid much attention to, after the prediction of the realization of a new type of ground state, i.e., a supersolid state in the hard core boson model ${ }^{8-10}$ and a pin-ball liquid state in the $t$ - $V$ model. ${ }^{11}$ In the former, most of the calculations have been performed by QMC simulation, and in the latter, ED has been performed. In QMC, it is difficult to calculate in parameter ranges $V / t \gtrsim 10$. For ED, the system size is limited. Thus, our proposal should be important to clarifying the features at certain parameter ranges or system sizes, at which previous methods cannot be applied.

We would like to thank M. Miyazaki and C. Hotta for stimulating discussions.

1) See, for example, F. Alet et al.: J. Phys. Soc. Jpn. Suppl. 74 (2005) 30, and A.F. Albuquerque et al.: J. Magn. Magn. Mater. 310 (2006) 1187.

2) S. Capponi and D. Poilblanc: Phys. Rev. B 66 (2002) 180503.

3) S. Capponi et al.: Phys. Rev. B 70 (2004) 104424.

4) G. H. Wannier: Phys. Rev. 79 (1950) 357.

5) I. Syozi: Prog. Theor. Phys. 6 (1951) 306.

6) K. Kano and S. Naya: Prog. Theor. Phys. 10 (1953) 158.

7) P. W. Anderson: Phys. Rev. 102 (1956) 1008.

8) S. Wessel and M. Troyer: Phys. Rev. Lett. 95 (2005) 127205.

9) D. Heidarian and K. Damle: Phys. Rev. Lett. 95 (2005) 127206.

10) R.G. Melko et al.: Phys. Rev. Lett. 95 (2005) 127207.

11) C. Hotta and N. Furukawa: Phys. Rev. B 74 (2006) 193107. 